\title{
SINGLE GRAIN ORIENTATION MEASUREMENTS APPLIED TO THE FORMATION AND GROWTH OF RECRYSTALLIZATION NUCLEI
}

\author{
O. ENGLER, C. ESCHER and G. GOTTSTEIN \\ Institut für Metallkunde und Metallphysik, RWTH Aachen, Kopernikusstr. 14, \\ D-52056 Aachen, Germany
}

(Received 18 December 1995)

\begin{abstract}
Measurements of the crystallographic orientation of individual grains provide information on the spatial arrangement and distribution of orientations in a crystalline solid, i.e. on the orientation topography, which can be utilized to establish a basis for a deeper understanding of the microstructural mechanisms during deformation and recrystallization of metallic materials.

Techniques for the convenient measurement of single grain orientations with high accuracy by evaluation of Kikuchi diagrams in a TEM and SEM (EBSD) are introduced. The principles of Kikuchi pattern formation as well as the measuring and evaluation procedures are outlined. Particular attention is focused on the automation of pattern indexing in order to provide fully automated evaluation routines. The unique potential of these techniques is demonstrated by two examples pertaining to the microstructural evolution during the formation of recrystallization nuclei and their subsequent growth.
\end{abstract}

KEY WORDS: SEM, TEM, EBSD, Kikuchi diagrams, Hough-transform, Radon-transform, microbands, SIBM, recrystallization, nucleation, growth selection.

\section{INTRODUCTION}

Recrystallization of deformed metallic material involves the nucleation and growth of new undeformed grains. With progressing recrystallization, these new grains grow at the expense of the surrounding deformed matrix by migration of high angle grain boundaries, which causes characteristic changes in the crystallographic texture. Hence, analysis of the recrystallization textures yields valuable information about the underlying mechanisms of recrystallization, especially on the formation and growth of the nuclei, as e.g. thoroughly discussed by Beck and Hu (1966).

Nowadays, microtexture investigations add a new element for a quantitative description of microstructure evolution during recrystallization (e.g. $\mathrm{Hu}, 1969$ ). Inasmuch as the macroscopic texture characterizes anisotropic behaviour of a polycrystalline aggregate, the local orientation distribution probes the mechanisms that govern microscopic structure development. In literature a variety of techniques for determination of single grain orientations have been reported, for overviews see e.g. Humphreys (1984), Gottstein (1992) and Schwarzer (1993). Although for determination of local orientations in the TEM frequently still selected area diffraction (SAD) patterns are evaluated, local orientations can be determined much more precisely by evaluation of the Kikuchi diagrams (Figure 1a) (v. Heimendahl et al., 1964). In the SEM, "Electron Back Scatter Diffraction" (EBSD; sometimes also called "Backscatter Kikuchi Diffraction", BKD) 


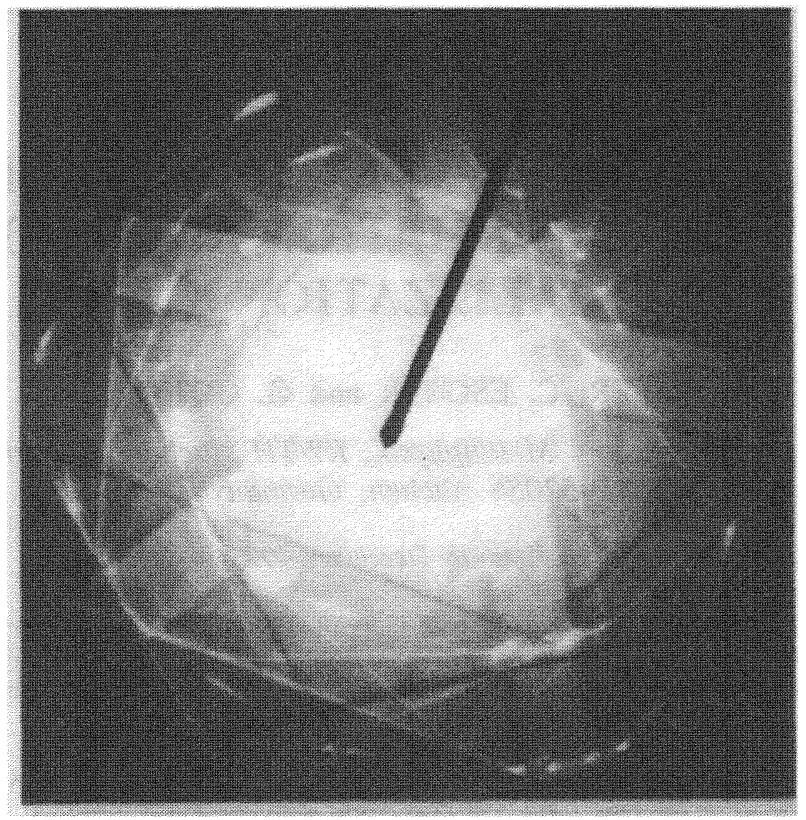

(a) TEM

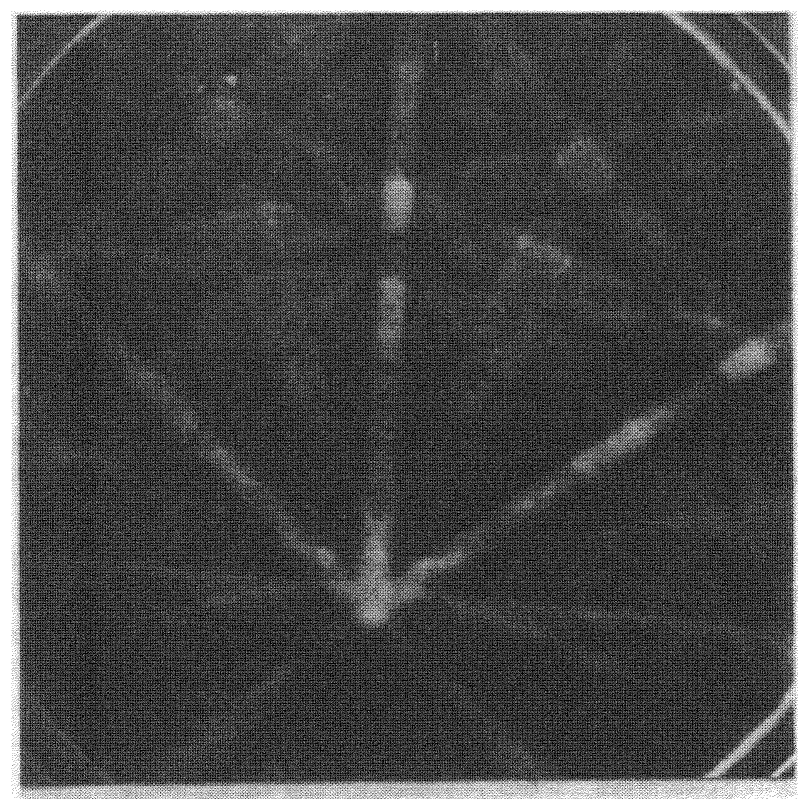

\section{(b) SEM (EBSD)}

Figure 1 Kikuchi diagrams. (a) TEM (partially recrystallized $\mathrm{Ni}_{3} \mathrm{Al}$ ), (b) SEM, EBSD (recrystallized $\mathrm{Al})$. 
is the obvious choice for the determination of single grain orientations (Figure $1 b$ ), owing to its ease of measurements combined with high accuracy and high spatial resolution. In the following, the principles of formation of Kikuchi diagrams both in the TEM and SEM will be briefly introduced. Furthermore, the possibilities of orientation determination and particularly of automated evaluation of Kikuchi diagrams will be addressed.

Commonly, the pursuit of microstructural evolution involves different dimensional scales, and it is important to use the proper tool for the considered microstructural scale in order to obtain the desired information. As much as optical microscopy and SEM can hardly reveal the mechanisms of nucleation of recrystallization, TEM fails to provide insight into the growth processes during advanced stages of recrystallization. Therefore, by application of complementary methods in the SEM and in the TEM to the same process at different microstructural scales, we gain much more valuable information than accessible by unrelated use of the individual techniques. This will be demonstrated by means of two examples concerning the evolution of microstructure and microtexture during the formation of recrystallization nuclei as well as their subsequent growth into the deformed matrix.

\section{ORIENTATION DETERMINATION BY ELECTRON DIFFRACTION}

\section{Formation of Kikuchi pattern in the TEM}

If one directs the electron beam in a TEM to thicker but still transparent regions of the sample, the typical SAD spot pattern attenuates and, instead, a so called Kikuchi pattern appears consisting of different sets of straight lines (Figure 1a). For a good spatial resolution of orientation determination the illuminated area must be small, which can be obtained by a finely focused electron beam illuminating only a very small area of the specimen (Micro Beam Electron Diffraction, MBED). Best spatial resolution can be obtained by convergent rather than parallel electron beam geometry, the respective diffraction mode will be referred to as Convergent Beam Electron Diffraction (CBED) in the following. In the latter case, Kikuchi patterns are obtained from thin sample areas as well.

As will be described in the following, the Kikuchi lines are directly related to the orientations of the reflecting planes in the specimen and, therefore, they are perfectly suited for crystal orientation determination. In order to understand the principles of orientation determination, the formation of Kikuchi lines can be explained in terms of a simplified model which only considers the geometrical aspects (Kikuchi, 1928). An electron beam entering a crystalline solid is subject to elastic and/or diffuse scattering (Figure 2). The elastic scattering gives rise to distinct diffraction spots in the back focal plane, which can be utilized for SAD. In case of diffuse scattering the atomic planes of a crystalline specimen are showered by electrons arriving from all directions, and for each set of planes there will always be some electrons that obey Bragg's law, i.e. they diffract at a specific angle $\theta$. These electrons emerge from the sample along the surface of a cone (Kossel-cone) which extends about the normal of the reflecting atomic planes with half apex angle $90^{\circ}-\theta$. Since the wavelength $\lambda$ of the electrons is very small, however, $\theta \approx \lambda / 2 d$ ( $d$ : interplanar spacing) is very small as well. Consequently, the apex angle of the diffraction cone is close to $180^{\circ}$, i.e. the line of interaction of the cone with the film plane is essentially straight (Figure 1a, 2). 


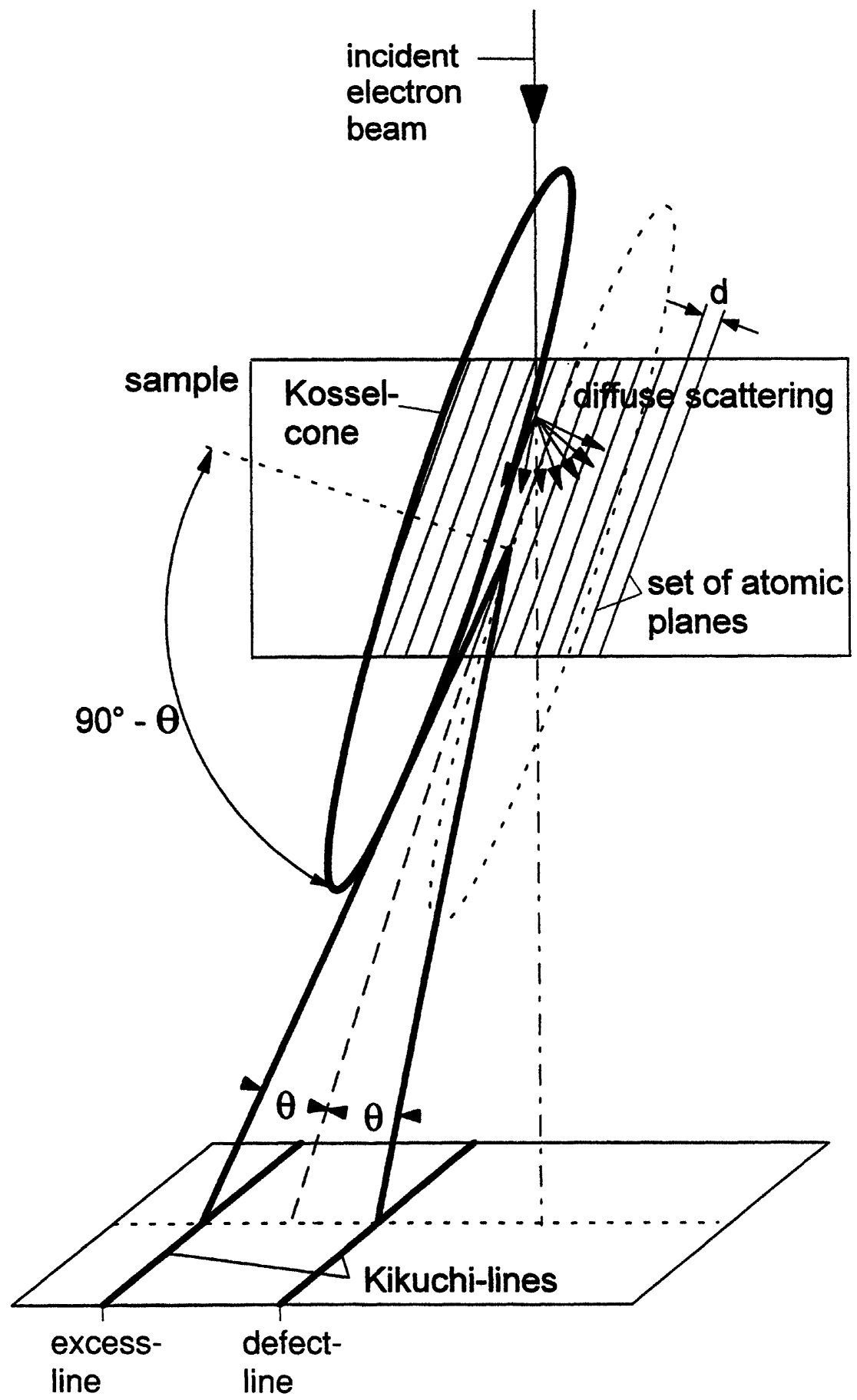

Figure 2 Formation of Kikuchi lines in the TEM. 
For each set of atomic planes two parallel Kikuchi lines with an angular distance of $2 \theta$ will form, which exhibit quite different intensities (Figure 2). In a very simplistic approach the two lines can be understood as follows: Generally, the angles between the inelastically scattered beam that impinges upon the crystal planes and the primary beam are not equal, which leads to a non-equal transfer of electrons along each cone surface. In the direction of the incident electron beam, where Bragg's law is satisfied, low-intensity lines (defect lines) will occur as there the electrons are scattered away and, thus, do not contribute to the transmitted intensity. Lines of higher intensity (excess lines) are obtained in the direction into which the electron beam is diffracted since there the electrons add to the background intensity. The reflecting plane would bisect the respective excess and defect Kikuchi lines in the film plane (Figure 2).

Hence, a Kikuchi pattern consists of bands with two parallel lines. Each band has a distinct width and corresponds to a distinct crystallographic plane. Accordingly, an intersection of bands represents a zone axis. By measuring the angles between several zones or zone axes the crystallographic orientation of the illuminated microscopic volume can readily be determined, as will be discussed in more detail in later sections.

For on-line measurements, the Kikuchi pattern is captured with a low light CCDcamera and then transferred through a video-grabber card to a computer for storage and orientation determination. The camera can be attached outside the TEM and record the pattern either from the (tilted) main viewing screen (Høier et al., 1994) or from an additional screen that has to be moved into the electron beam (Zaefferer and Schwarzer, 1994a). In another set-up the camera is mounted below the main viewing screen (Weiland, 1994). In our arrangement, the chip of a Peltier-cooled slow scan CCD-camera (model Gatan 689) is inserted into the electron beam slightly above the main viewing screen of the TEM (JEM 2000 FX, JEOL; see also Krieger Lassen, 1995).

\section{Electron diffraction in the SEM}

EBSD in a SEM was first developed by Venables and Harland (1973) but improved for on-line orientation determination by Dingley et al. $(1984,1992)$ and Hjelen et al. (1993) since. The principles of pattern formation from backscattered electrons in the SEM are akin to those in the TEM described above. In contrast to the sharp transmission Kikuchi lines, however, the backscattered EBSD patterns in a SEM consist of bands of higher intensity (Pseudo Kikuchi lines, Figures 1b, 3) rather than sharp Kikuchi lines, which is caused by the different electron transfer functions in transmission and back reflection.

A schematic set-up for analysing EBSD patterns in the SEM is shown in Figure 4. To obtain an EBSD pattern the electron beam is focused on to the position of interest in a specimen. The sample is strongly tilted $\left(60^{\circ}-75^{\circ}\right)$ for maximum gain of backscattered electrons. The pattern is revealed on a phosphorus screen placed in the vacuum chamber of the SEM (JSM 6100, JEOL) and is recorded with a low light SITcamera through a lead glass window from outside the SEM. The pattern is then processed by an image processor (ARGUS 10, Hamamatsu) to improve pattern quality and, similarly as already described for the TEM, finally transferred to a personal computer for storage and orientation determination.

Best pattern contrast is obtained at high acceleration voltage (typically $20 \mathrm{kV}$ ) and high beam current $(1-100 \mathrm{nA})$. Sample preparation is usually easy and can readily be carried out with standard metallographical methods. For sufficient pattern quality, 


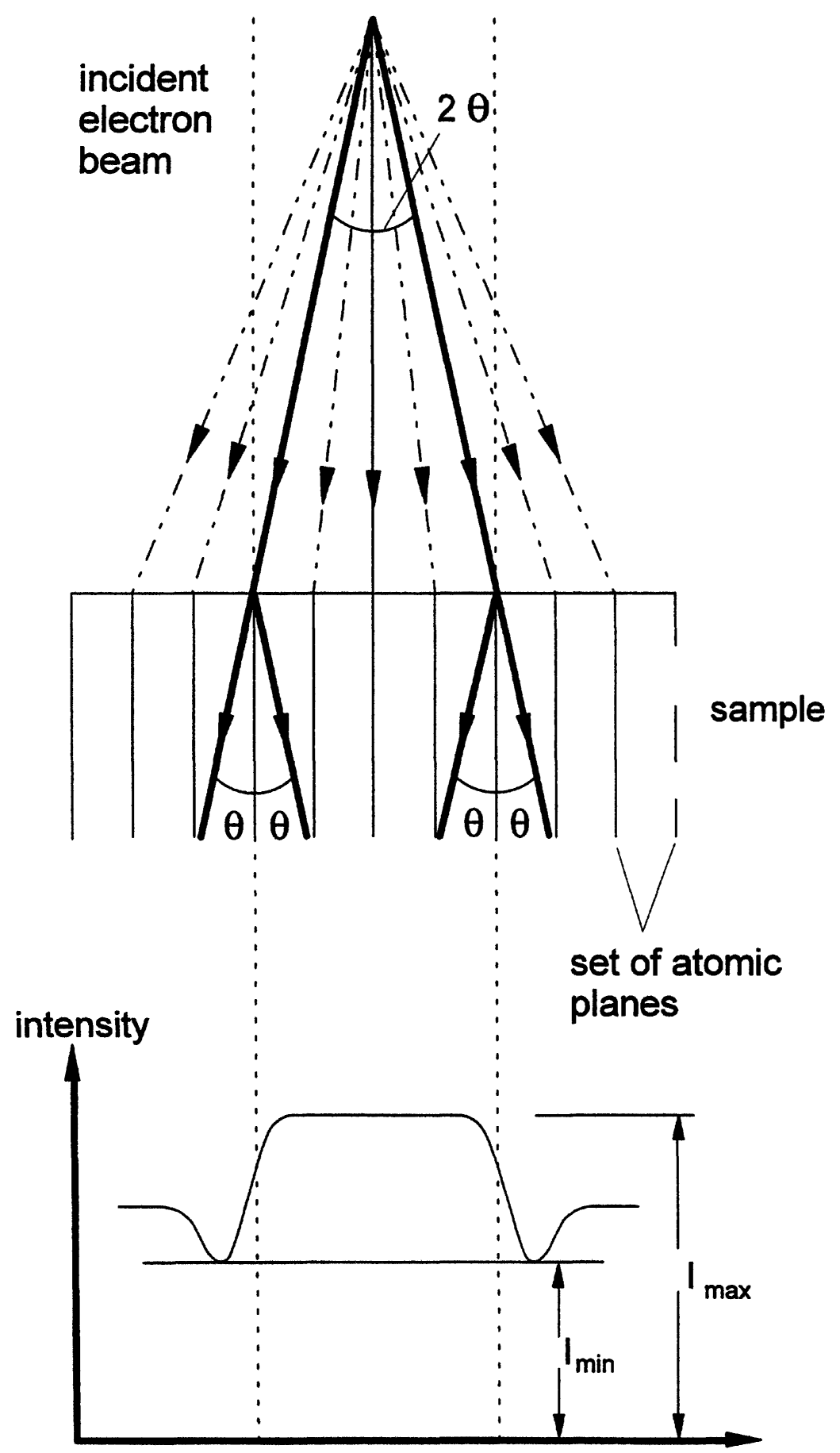

Figure 3 Formation of pseudo Kikuchi bands in the SEM. 


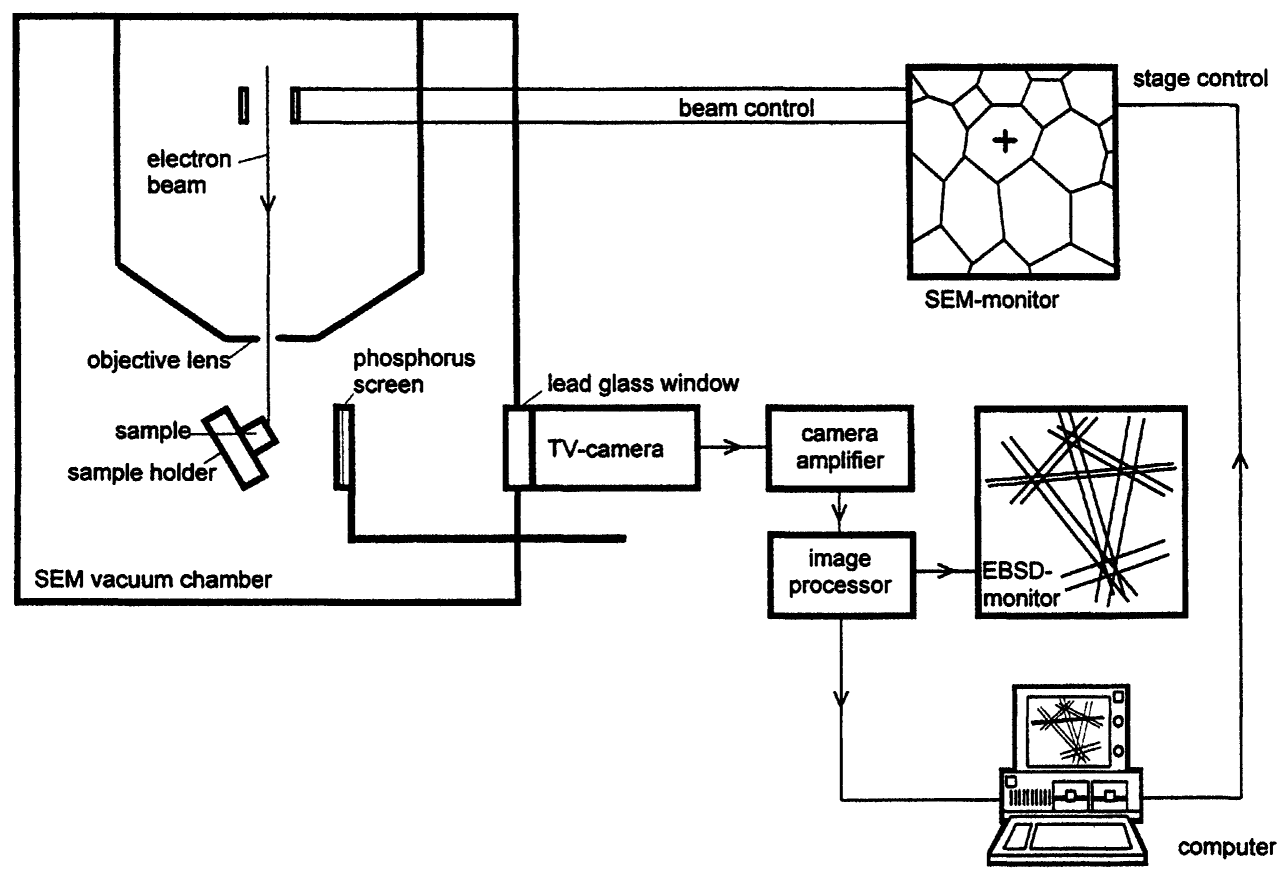

Figure 4 Scheme of a set-up for automated EBSD analysis.

however, the sample surface must not show any deformation. Thus electrolytical polishing or slight chemical etching commonly yield best results.

\section{Orientation determination from Kikuchi patterns}

In order to determine the orientation of the illuminated volume of the sample, first the Kikuchi pattern has to be indexed. For an interactive orientation evaluation, the screen coordinates of at least three bands or axes in the pattern are marked by the user with a computer mouse (algorithms for an automatic pattern recognition will be described in the next section). With an appropriate calibration of the hardware set-up, which is described e.g. by Hjelen et al. (1993) and Krieger Lassen and Bilde-Sørensen (1993), the angles between the bands or zone axes can be determined and compared to a look-up table that contains all angle combinations possible in the corresponding crystal structure. Some small measuring uncertainty (typically $1^{\circ}-2^{\circ}$ ) must be tolerated, of course. Moreover, the width of the bands is related to the Bragg angle (i.e. 2 $\theta$, see above), which can also be utilized to index the bands or to check the indexing. For EBSD, however, the measurement of the band width is not very accurate owing to the low signal-to-noise ratio of the pattern and, therefore, the band widths are usually not considered for orientation determination.

After indexing the pattern, the orientation of the illuminated crystal volume with respect to the laboratory coordinate system can be determined (cf. Dingley et al. 1987, Schmidt et al., 1991, Wright and Adams, 1992, Engler and Gottstein, 1992, Zaefferer 
and Schwarzer, 1994b). The crystallographic orientation is usually given in terms of its orientation matrix $g$, which transforms the sample coordinate system (indexed by the superscript $S$ in the following) into the coordinate system of the crystal (superscript C).

After the indexing, for each vector $r_{i}$ (axis or band) its crystallographic direction $r_{i}^{c}=\left(x_{i}^{c}, y_{i}^{c}, z_{i}^{c}\right)$ as well as its vector in the sample frame $r_{i}^{s}=\left(x_{i}^{s}, y_{i}^{s}, z_{i}^{s}\right)$ is known. For non-cubic crystal structures the crystallographic vectors $r_{i}^{c}$ have to be transformed into an orthonormal coordinate system by multiplication with the inverse crystal matrix (Young and Lytton, 1972), but then they can equally well be examined. It follows from the definition of $g$ (with $i \in 1,2,3$ ):

$$
\left(\begin{array}{lll}
x_{1}^{c} & x_{2}^{c} & x_{3}^{c} \\
y_{1}^{c} & y_{2}^{c} & y_{3}^{c} \\
z_{1}^{c} & z_{2}^{c} & z_{3}^{c}
\end{array}\right)=g \cdot\left(\begin{array}{lll}
x_{1}^{s} & x_{2}^{s} & x_{3}^{s} \\
y_{1}^{s} & y_{2}^{s} & y_{3}^{s} \\
z_{1}^{s} & z_{2}^{s} & z_{3}^{s}
\end{array}\right) \quad \text { or } g=\left(\begin{array}{lll}
x_{1}^{c} & x_{2}^{c} & x_{3}^{c} \\
y_{1}^{c} & y_{2}^{c} & y_{3}^{c} \\
z_{1}^{c} & z_{2}^{c} & z_{3}^{c}
\end{array}\right) \cdot\left(\begin{array}{lll}
x_{1}^{s} & x_{2}^{s} & x_{3}^{s} \\
y_{1}^{s} & y_{2}^{s} & y_{3}^{s} \\
z_{1}^{s} & z_{2}^{s} & z_{3}^{s}
\end{array}\right)^{-1}
$$

For computation of the orientation matrix $g$ the second matrix in Eq. (1) must be inverted, which requires its determinant to be different from zero (i.e. the matrix must be regular). Matrix inversion is much easier for orthogonal matrices, where $A^{-1}=A^{T}$, so that only the rows and lines of the matrix must be exchanged. In order to obtain an orthogonal matrix, 3 new vectors $\tilde{r}_{i}^{s}$ in the sample frame and $\tilde{r}_{i}^{c}$ in the crystallographic frame are formed according to:

$$
\tilde{r}_{1}^{s}=r_{1}^{s} \times r_{2}^{s}, \tilde{r}_{2}^{s}=\tilde{r}_{1}^{s} \times r_{3}^{s}, \tilde{r}_{3}^{s}=\tilde{r}_{1}^{s} \times \tilde{r}_{2}^{s}
$$

and, correspondingly:

$$
\tilde{r}_{1}^{c}=r_{1}^{c} \times r_{2}^{c}, \tilde{r}_{2}^{c}=\tilde{r}_{1}^{c} \times r_{3}^{c}, \tilde{r}_{3}^{c}=\tilde{r}_{1}^{c} \times \tilde{r}_{2}^{c}
$$

An easier orthogonal arrangement can be gained just by setting $\tilde{r}_{1}^{s}=r_{1}^{s}$ and correspondingly combining the other vectors, as proposed by Wright et al. (1992). In that case two bands or axes are sufficient to compute $g$. However, with only two vectors indexing is never unequivocal, and moreover, the use of three vectors introduces some averaging during the computation of $g$.

In order to avoid wrong solutions and to distinguish between $[u v w]$ and $[\bar{u} \bar{v} \bar{w}]$ directions, a pattern is recalculated from the orientation $g$ and compared with the original pattern. From the orientation matrix $g$ the Miller indices, Euler angles or Rodrigues vectors can readily be computed according to standard algorithms and can be stored in a computer file for further evaluation and representation of the data (e.g. Randle, 1992, Gerth and Schwarzer, 1993, Engler et al., 1994).

\section{Automation of single grain orientation measurements}

With an interactive procedure as described above about $50-100$ orientation can be evaluated in an hour. For improvement of performance the time consuming manual marking of zones or axes has to be replaced by an automatic indexing code. In particular, for fully automated systems as necessary for orientation imaging microscopy (OIM, Adams et al., 1993) automatic pattern recognition is essential. This requires that the entire pattern information recorded by the camera has to be transferred pixel by pixel to a computer by means of a video grabber card. There, the position of the bands or poles is detected automatically by a suited pattern recognition code. Subsequently, the 
pattern can be indexed and the crystallographic orientation can be computed as described in the last section. In early attempts of automatic pattern recognition it was tried to directly detect the position of the zone axes (Wright et al., 1991) or the bands (Juul Jensen and Schmidt, 1990) in the EBSD pattern. More recently, more advanced algorithms of pattern recognition have been employed and the former methods are no longer competitive today. Wright and Adams (1992) tried to detect the band edges from the local intensity gradients in the pattern by means of the Burns-algorithm. Krieger Lassen et al. (1992) and Kunze et al. (1993) transformed the EBSD pattern by means of the Hough-transform (or Radon-transform, see below), as applied to the automatic evaluation of Laue patterns already by Gottstein (1988). Nowadays, codes for the automatic indexing of TEM Kikuchi diagrams by means of the Hough-transform have been developed as well (Zaefferer and Schwarzer, 1994a, Krieger Lassen, 1995). By this procedure lines in the original image are transformed into points, which can be detected much easier by computer codes. A careful comparison of these two pattern indexing routines showed that both the Burns- and the Hough-algorithms seem to be equally reliable, although both algorithms have their advantages (Kunze et al., 1993). In general, for poorer pattern quality the Hough-transform yields slightly more accurate results, whereas the Burns algorithms is advantageous for high quality patterns. Furthermore, the velocity of the Hough-transform does not depend on the pattern quality, which favours it for automated systems.

In the following, the approach which was implemented at the Institut für Metallkunde und Metallphysik in Aachen for evaluation of EBSD patterns shall briefly be introduced. Subsequently, the computer code which was adapted for Kikuchi patterns in the TEM will be addressed.

First, several steps of pre-processing of the original pattern are taken to obtain optimum conditions for the subsequent Hough-transform. The original EBSD pattern (e.g. Figure $1 b$ ) is substantially reduced to $101 \times 101$ pixels by averaging over $5 \times 5$ pixel blocks (Figure 5a) for cutting down on computation time. Subsequently, contrast enhancement of the pattern is performed by linear scaling of the grey values $I(x, y)$ to the entire spectrum $0 \leq I(x, y) \leq \cdot 255$.

The EBSD patterns contain a large amount of background intensity as well as variations in the level of background intensity (cf. Figure $1 \mathrm{~b}$ ). Thus, the next step of image processing is to correct the pattern with respect to the background intensity and, simultaneously, to shading differences. The pattern background is determined by convoluting the image with a low pass filter, whose size $(10 \times 10$ pixels $)$ is chosen so as to reduce shading differences most effectively without loosing pattern information. After bilinear extrapolation the background image $B(x, y)$ is obtained and subtracted from the original image $I^{*}(x, y)=I(x, y)-B(x, y)$. This procedure is repeated several times so as to yield a smooth background that is necessary for the subsequent image transformation. Figure $5 \mathrm{~b}$ shows the pattern of Figure $5 \mathrm{a}$ after the pre-processing.

The next step of the procedure is the transformation of the pattern. During the Houghtransform (Hough, 1962), each point $\left(x_{i}, y_{i}\right)$ of the original image is transformed into a sinusoidal curve in the Hough-space, which is formed by the coordinates $\rho$ and $\varphi$ according to the relation:

$$
\rho(\varphi)=x_{i} \cdot \cos \varphi+y_{i} \cdot \sin \varphi
$$



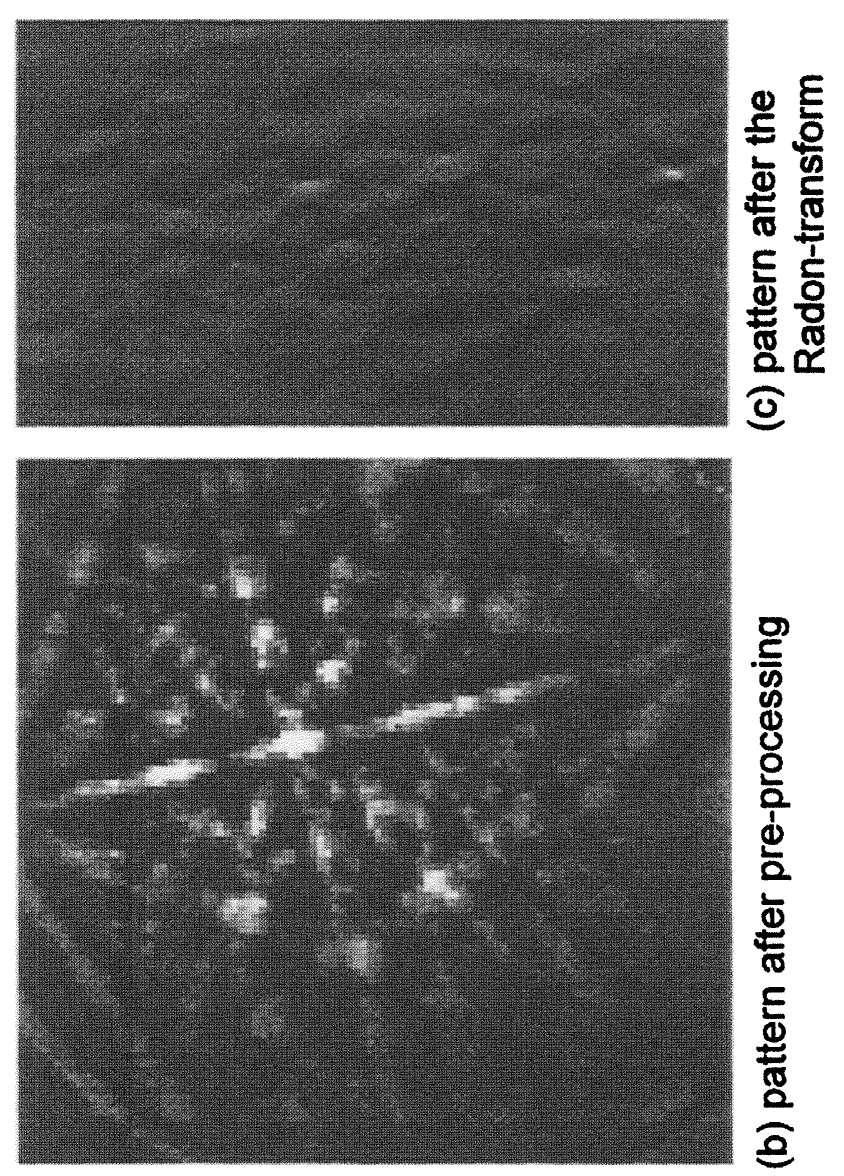

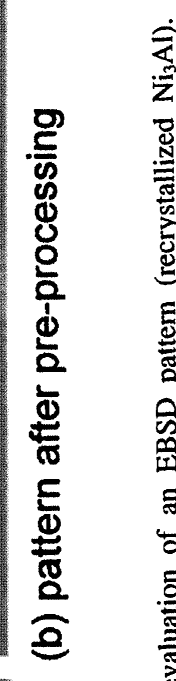

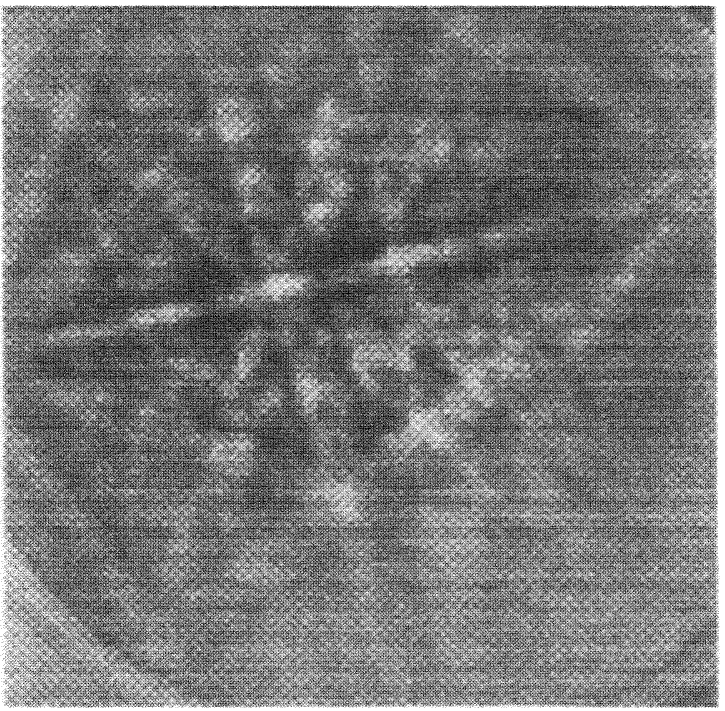

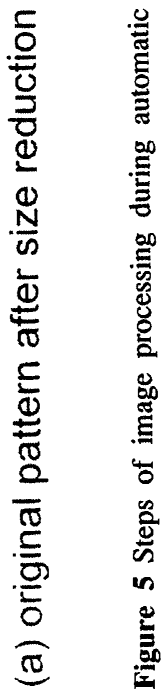


This relation specifies a line by the angle $\varphi$ of its normal and its distance $\rho$ from the origin (here, the centre of the image). Points which lie on a line in the original image (Figure 6a) have the same distance $\rho$ and angle $\varphi$ with respect to the origin and, hence, they all accumulate in one and the same point in Hough-space (Figure $6 b)$.

For a transformation of a grey-tone image as an EBSD pattern, each point of the original image $\left(x_{i}, y_{i}\right)$ is transformed into the Hough-space $(\rho, \varphi)$ under consideration of its intensity $I\left(x_{i}, y_{i}\right)$, a procedure which is referred to as grey-tone weighted Houghtransform or Radon-transform (Voss and Süße, 1991). For obtaining a homogeneously transformed image without shading effects, only a circle with a diameter of 101 pixels is considered during the transform rather than the entire square pattern. In Figure 5c, the Radon-transformed image of Figure $5 b$ is represented.

The next step is the identification of the peaks in Radon-space, i.e. the bands in the original image. In the transformed image, the peaks have a characteristic butterflylike shape (Krieger Lassen et al., 1992). Hence, the peaks can be detected by convolution of the Radon-space with a filter which has the typical butterfly shape. Application of this filter strongly enhances the centre of the peaks in $\varphi$-direction and so the angle $\varphi$ of the bands can be detected with high accuracy. For detection of the $\rho$-value of the peaks, i.e. the exact position of the mid of the EBSD bands, a multitude of different peaks has been analysed to yield an average "standard-profile" of the peaks in the $\rho$-direction, and so, the $\rho$-coordinate of the bands in the Radon-space can be reliably determined.

The detected bands are listed according to their relative intensity, and the five bands with the highest intensities are used for further orientation computation as described in the previous section. Occasionally wrong lines are detected and, consequently, no solution is obtained and the operator has to manually reject the wrong lines. In other cases ambiguous solutions are found, particularly in the presence of symmetrical poles. In that instance, all possible solutions are listed and the user has to select the right one, e.g. by input of additional lines. For a fully automated system, i.e. for orientation microscopy (see below), interaction by the user is not possible, of course. Therefore, the detection algorithm had to be improved to yield unique solutions in as many cases as possible. An automatic permutation of the detected bands under consideration of less intense bands yielded very promising results by strongly reducing the number of non-uniquely indexed patterns. During a critical evaluation of this algorithm it appeared that more than $95 \%$ of the patterns, of course strongly depending on the pattern quality, are unambiguously and accurately indexed. With regard to the accuracy of the automatic indexing routine, about 200 orientations were evaluated both manually and by the automatic indexing routine. Comparison of the obtained data yielded ar average misorientation of $1-2^{\circ}$, i.e. no noticeable difference to the relative error of EBSD measurements (see below). With a computation time for the entire algorithm of less than $4 \mathrm{~s}(486 \mathrm{PC}, 50 \mathrm{MHz})$ the automatic indexing routine works very conveniently, with high velocity and, particularly, with high reliability.

As discussed above, the TEM Kikuchi diagrams underlie the same physical and crystallographic principles as the EBSD patterns in the SEM. Therefore, based on the success of automatic indexing of EBSD patterns, the computer code was also adapted for evaluation of Kikuchi patterns in the TEM. However, for the TEM Kikuchi diagrams more serious problems had to be overcome for an automated pattern recognition, and the computer code had to be substantially modified to yield satisfactory results. For the TEM diagrams, the CCD-camera yields a slightly different image size $576 \times 384$ 


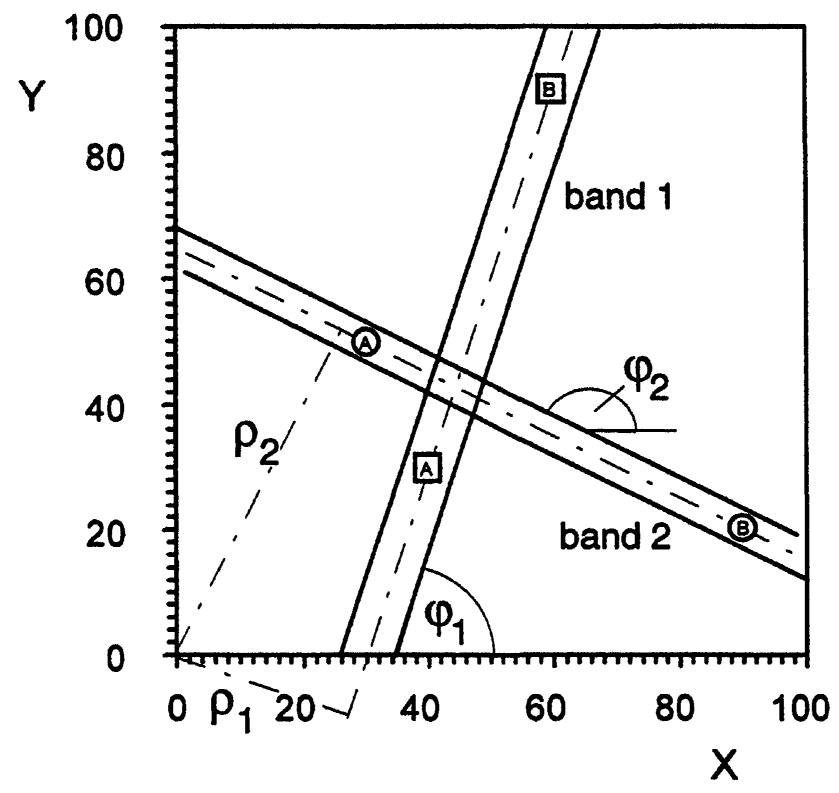

(a) original image

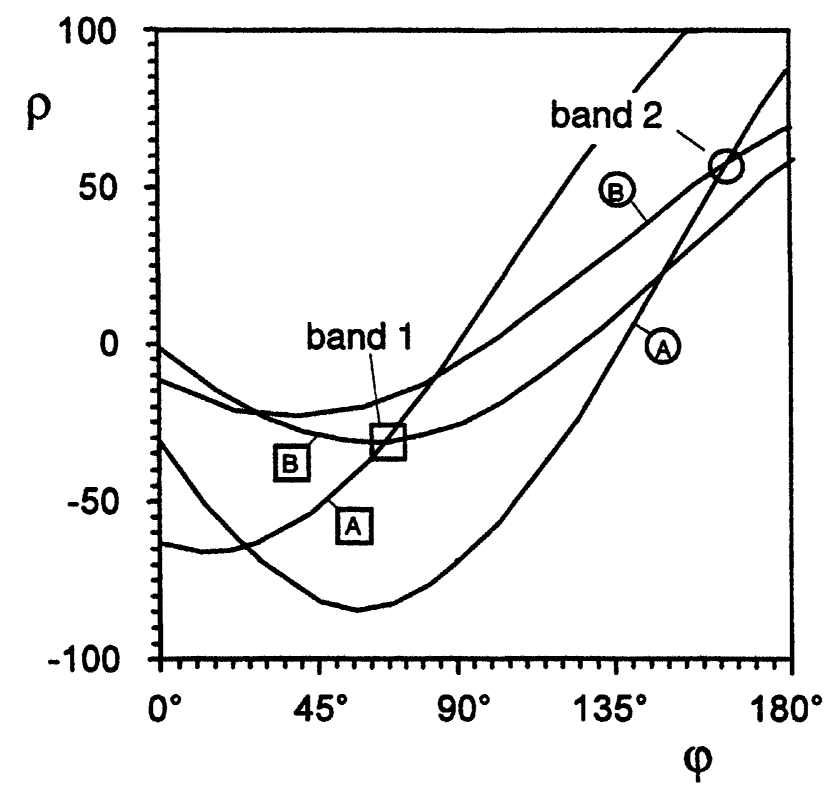

(b) Hough-space

Figure 6 Schematic representation of the Hough-transform (a) two bands in the original image; and (b) bands of (a) in the Hough-space. 
pixels, which is reduced by averaging over $4 \times 4$ pixel blocks. In comparison to EBSD patterns additional steps of image processing are required, since the Kikuchi patterns are frequently superimposed by high intensity SAD-diffraction spots and, particularly, by the primary beam, whose intensity may exceed that of the pattern by several orders of magnitude. First, the pattern is convoluted by a "sharpen filter" to enhance the contrast of the Kikuchi lines. Then, localized high intensities are replaced by the average value of their neighbourhood, since these regions most likely correspond to the SAD-spots. The area affected by the primary beam spot is simply neglected for the Hough-transform. Owing to the smaller steric angle, but a larger number of visible high-index bands, unambiguous indexing is more complicated than for EBSD. On the other hand, the determination of the band width is sufficiently accurate so as to check the band indexing, which is utilized to identify wrong solutions.

Further automation of large scale single grain orientation measurements requires automated scanning of the selected specimen area with a computer controlled sample stage (Orientation Imaging Microscopy, "OIM", Adams et al., 1993). Rather than moving the sample, it is also possible to control the electron beam in the SEM so as to scan the sample surface (Schwarzer et al., 1995). In our system, the SEM has been equipped with a stage control (Sprite HR2, Deben Research) which enables the computer to move the samples in steps of minimum $1 \mu \mathrm{m}$. Therewith, the entire EBSD set-up, i.e. stage control, pattern recognition and data evaluation, has eventually been automated. With a sampling rate exceeding 600 orientations per hour large area orientation topography can be obtained and orientation microscopy can be performed. From the misorientations between adjacent measurement points, grain boundaries can be revealed and classified according to their character. So, large angle grain boundaries, small angle grain boundaries and subgrains can be identified and evaluated with regard to morphology, orientation correlation, grain boundary character etc. If one further considers the quality of the pattern (e.g. Krieger Lassen et al., 1994), one can gain much more information on the microtexture and microstructure than accessible by standard metallographic techniques.

\section{Assessment of the methods for local orientation determination}

The main advantage of the TEM is obviously its excellent spatial resolution, which is at least one order of magnitude better than for the SEM. With CBED, Kikuchi diagrams from regions as small as a few nanometers can be obtained. This high spatial resolution also allows to investigate orientations in highly deformed samples as the level of lattice distortion is of minor importance. Finally, the TEM offers much more additional information on the microstructure, i.e. precipitates, dislocations and dislocation arrangements etc., than possible to obtain in the SEM. The major disadvantages of the TEM are, however, the difficult and laborious sample preparation and the very small area of view, which is usually much less than $100 \mu \mathrm{m}$ in diameter. This generally restricts the application of the TEM to few selected examples with only limited information on the statistical relevance of the effects observed.

Concerning the accuracy of orientation determination in the TEM, the relative error, i.e. the error in misorientation between two neighboured grains, is very low, i.e. less than $0.5^{\circ}$. For the absolute error higher values must be accepted, which are primarily caused by problems with calibration of the entire set-up and the sample mounting. The absolute error can be estimated to about $5^{\circ}$. 
For orientation determination in bulk specimens in the SEM, EBSD is best suited owing to its ease of performance combined with high accuracy and high spatial resolution. The evaluation of the patterns is very much facilitated by the large steric angle obtained, which speeds up evaluation and reduces ambiguity of the determined orientation. With regard to the spatial resolution, patterns have been recorded from volumes as small as $20 \times 80 \times 10 \mathrm{~nm}^{3}$ using a field emission gun (Harland et al., 1981). With standard set-ups, a spatial resolution of $250 \times 700 \mathrm{~nm}^{2}$ can be obtained (Hjelen and Nes, 1990), which also allows to investigate deformed samples, if the microstructure is comprised of undistorted (dislocation-free) volumes (i.e. cells or subgrains) in excess of this size. Owing to the lower signal-to-noise ratio of the EBSD pattern, the accuracy of orientation determination is somewhat smaller compared to TEM. With an accurate calibration a relative error of about $1-2^{\circ}$ can be obtained, which is sufficient for most texture applications, however.

\section{APPLICATIONS}

\section{Recrystallization nucleation in cold rolled $\mathrm{Ni} \mathrm{i}_{3} \mathrm{Al}+\mathrm{B}$}

The first example pertains to the nucleation of recrystallization in cold rolled polycrystalline boron doped $\mathrm{Ni}_{3} \mathrm{Al}$ (IC15). In an earlier investigation on this material, recrystallized grains were observed to form at grain boundaries, microbands and, at high strains, at shear bands (Ball and Gottstein, 1993a). In order to elucidate the incipient stages of nucleation, in-situ annealing experiments in the TEM were performed and the orientations of newly forming grains and the surrounding matrix were determined by MBED. The intermetallic compound $\mathrm{Ni}_{3} \mathrm{Al}$ is very well suited for this purpose, because neither cell formation nor recovery were observed prior to the onset of recrystallization and the high stored energy of the material results in a small critical nucleus size. Hence, the nucleation process can be observed within the transparent areas of the TEM samples (Escher and Gottstein, 1994). For the in-situ annealing experiments, thin foils were prepared from the longitudinal section of the samples and mounted onto a hot stage in the TEM. Details of sample processing and foil preparation are given elsewhere (Ball and Gottstein, 1993b). During in-situ experiments, the possibility of a fast on-line orientation determination helps to track orientation changes during the annealing process, as the development of new orientations can easily be recorded and the further evolution of the respective grains can be traced.

The first micrograph was taken from an in-situ experiment on a $10 \%$ cold rolled sample (Figure 7). In this sample nucleation of recrystallization at the former high angle grain boundaries was observed to prevail. In the example, after an annealing treatment of $150 \mathrm{~min}$ at $600^{\circ} \mathrm{C}$ and some minutes at $700^{\circ} \mathrm{C}$ the grain boundary started to bulge and tilting of the specimen exposed contrast at the tip of the bulges (Figure 7a). The generation of new grains at a former high angle grain boundary is facilitated by the difference in stored energy in the two grains and the good mobility of such boundary (Bailey, 1960). Orientation measurements were carried out and twin relationships were found between the tips ( $\mathbf{1}$ and $\mathbf{1}^{\prime}$ ) and the parent grain $\mathbf{A}$. The determined orientations are presented in a $\{111\}$ pole figure in Figure $7 \mathrm{~b}$, and the common $\langle 111\rangle$ axis of the twin relation is encircled. This example gives also evidence of an additional benefit of the TEM investigations: the possibility of specimen tilt. The tilting procedure permits the detection of contrast changes and thus makes the discovery of new orientations more easy. 


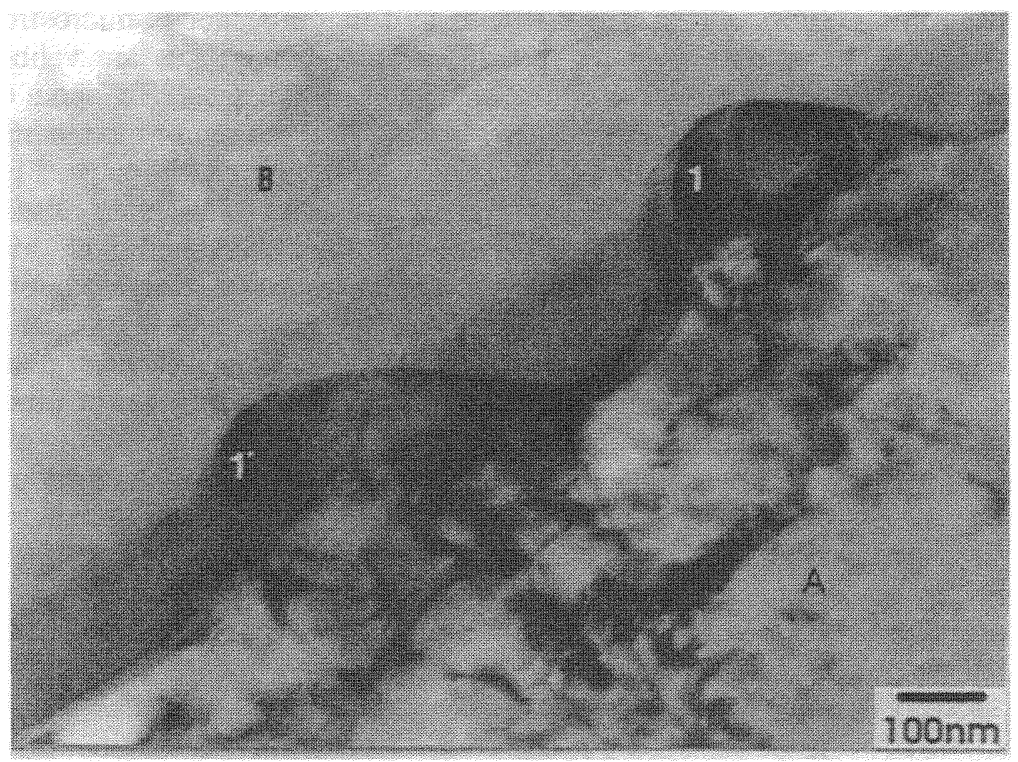

(a)

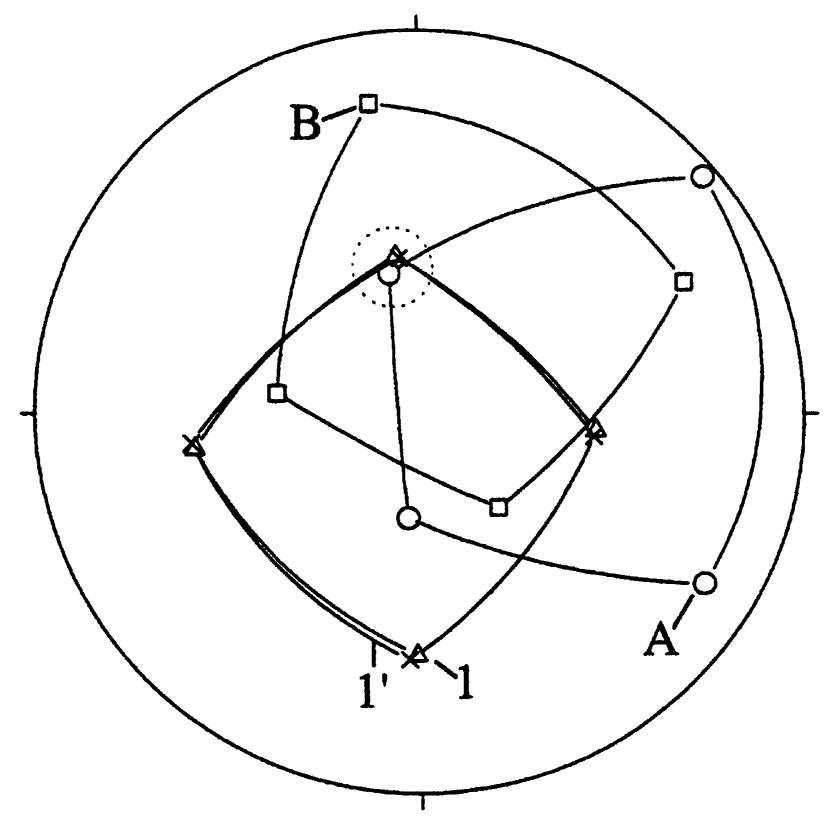

(b)

Figure 7 (a) Grain boundary bulging and generation of new orientations in a $10 \%$ cold rolled sample, (b) $\{111\}$ pole figure of the determined orientations. 
At higher rolling deformations $\mathrm{Ni}_{3} \mathrm{Al}$ develops an inhomogeneous microstructure. In the $40 \%$ cold rolled sample microbands of differently oriented sets are visible, which have been attributed to forced planar slip on $\{111\}$ slip planes (Ball and Gottstein, 1993b). During annealing, in addition to nucleation at grain boundaries new recrystallized grains emerged at these microbands, an example is shown in Figure 8a. In this instance, the temperature was slowly increased to $400^{\circ} \mathrm{C}$ and then raised in steps of $50^{\circ} \mathrm{C}$ up to $600^{\circ} \mathrm{C}$, with a $30 \mathrm{~min}$ hold at every temperature level. The orientation measurements and the calculation of the orientation relationships revealed approximate twin relationships between the matrix $\mathbf{M}$ and grain $\mathbf{1}$, between grain $\mathbf{1}$ and grain 2 and between grain 4 and grain 5 . Moreover, grain 2 and grain 4 were related by a $\Sigma 27 \mathrm{~b}$ misorientation, i.e. a third order twin relation. Deformation inhomogeneities like transition bands and microbands are known to be preferred sites for the nucleation of recrystallization ( $\mathrm{Hu}, 1963)$. Nucleation at a microband is assumed to be the result of higher stored energy within the very fine band in comparison to the matrix. Also, the forced planar slip may lead to an orientation gradient in the microband/matrix interface, which would favour the nucleation process as well. However, the new recrystallized grains did not grow very large, rather growth seemed to cease either by interference with other new grains or by twinning.

In the above examples irrespective of the nucleation site, grain boundaries or microbands, twin relationships were observed already at the very beginning of grain generation. This leads to the conclusion that twinning occurs easily and that the formation of twin boundaries plays a vital role in the nucleation process of the intermetallic compound $\mathrm{Ni}_{3} \mathrm{Al}$.

\section{Formation of the $R$-orientation in cold rolled and recrystallized Al-alloys}

The second example is to demonstrate the application of EBSD investigations to investigate the growth behaviour of recrystallization nuclei in Al-alloys. Besides the cube-orientation $\{001\}<100\rangle$, the recrystallization textures of many cold rolled Al-alloys comprise the $\mathrm{R}$-orientation $\{124\}<211>$, which is very similar to the rolling texture $\mathrm{S}$-orientation $\{123\}<634>$. In particular in the presence of $\mathrm{Fe}$, the R-orientation can grow up to a volume fraction of as much as $60 \%$, as shown e.g. by Ito et al. (1983) in high purity binary Al-Fe alloys and by Engler et al. (1996) in Al of commercial purity (AA1145). It has already been concluded by Beck and $\mathrm{Hu}$ (1952) that, owing to their similarity with the rolling texture S-orientation, $\mathrm{R}$-oriented nuclei can form in the deformed microstructure by two mechanisms: (i) The R-orientation can develop from the rolling texture by extended recovery reactions, i.e. by continuous recrystallization. This mechanism appears to be favoured in case of a strong hindrance of grain boundary motion by precipitates, but is of minor importance here. (ii) R-oriented grains may form by genuine, discontinuous recrystallization by nucleation at the grain boundaries between the deformed bands within S-oriented grains.

In the regions close to the grain boundaries generally higher dislocation densities and stronger orientation gradients exist due to the activation of additional slip systems to reduce strain incompatibilities at the grain boundaries during deformation. Therefore, best conditions for recovery and, consequently, for successful nucleation events prevail in regions close to the grain boundaries. Furthermore, the already existing large angle grain boundary between the two neighbouring grains ensures a good growth prospect of the nuclei. In Al-alloys, which usually comprise a recovered subgrain structure prior to the onset of recrystallization, nucleation at grain boundaries can proceed by growth 


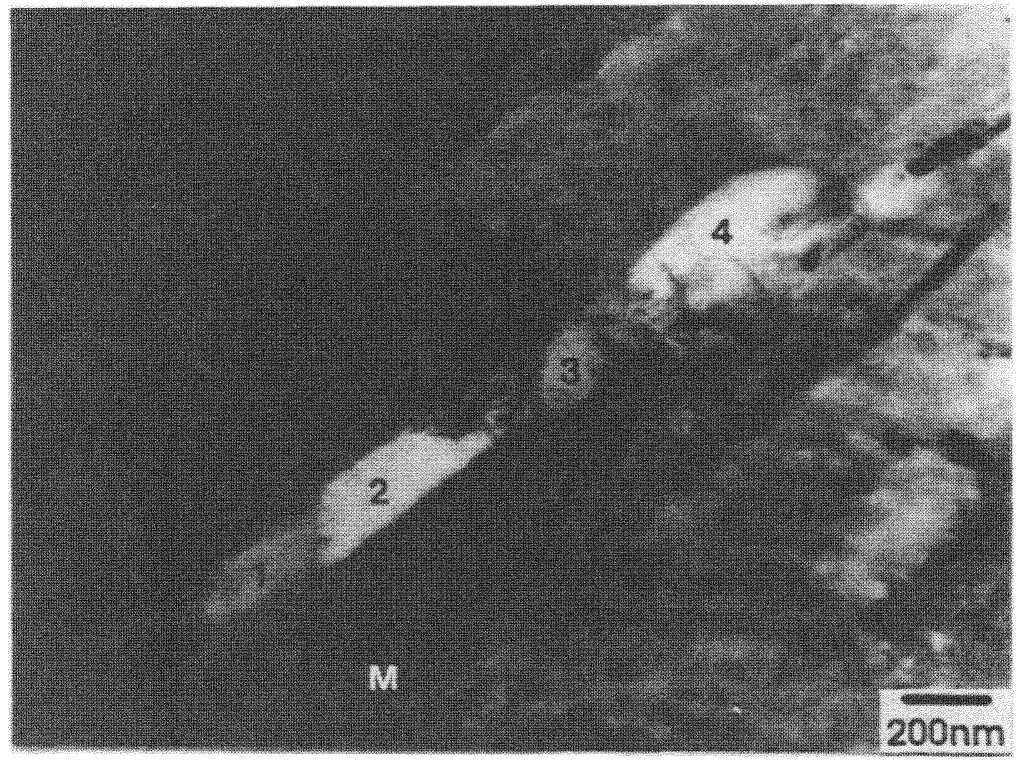

(a)

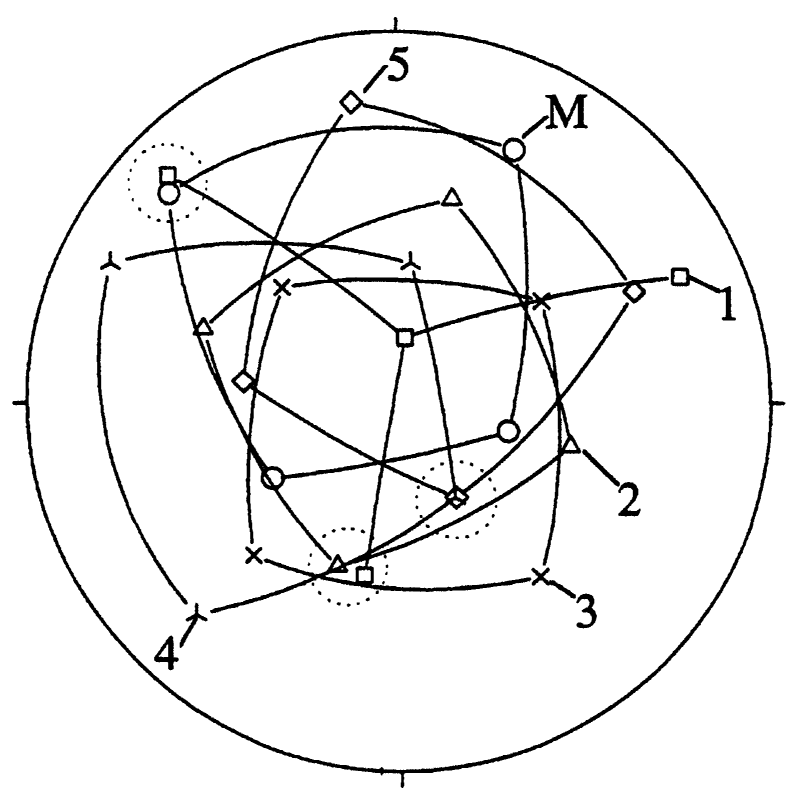

(b)

Figure 8 (a) Nucleation of new grains at a microband in a $40 \%$ cold rolled sample, (b) $\{111\}$ pole figure of the determined orientations. 
of a given subgrain on one side of the boundary into the deformed structure on the other side of the boundary (Bellier and Doherty, 1977). Thus, this mechanism which is generally referred to as "strain induced boundary migration" (SIBM) should lead to the formation of grains with orientations close to those that are already present in the deformation texture. Consequently, it can be anticipated that the R-oriented grains in recrystallized Al-sheets form through SIBM.

However, a direct analysis of the evolution of R-oriented grains from the state of nucleation until the final recrystallized structure was not possible prior to the emergence of techniques for individual grain orientation measurements in the SEM. Bellier and Doherty (1977) and Dadson and Doherty (1992) utilized the transmission Kossel technique in a SEM to study the formation of grains that had formed through SIBM in $\mathrm{Al}$ after uni-axial compression to medium strains $(\leq 40 \%)$. They observed that SIBM was the prevailing nucleation mechanism, and that the orientations of these grains were related to their respective parent grains. However, owing to the limitation of the spatial resolution of the Kossel technique to 5-10 $\mu \mathrm{m}$, only coarse grained samples could be investigated. Nowadays, such experiments can also be performed on fine grained material, e.g. commercial grades, by means of EBSD with its much higher spatial resolution better than $1 \mu \mathrm{m}$.

Figure 9 shows an example of several new recrystallized grains, which apparently nucleated at a former grain boundary in commercially pure Al (AA1145); a schematic sketch of the situation is given in Figure $9 \mathrm{~b}$. The sample was annealed for $1000 \mathrm{~s}$ at $250^{\circ} \mathrm{C}$ so as to initiate recrystallization. The example depicts a grain boundary separating two deformed bands $\mathbf{A}$ and $\mathbf{B}$ which comprise an approximate S-orientation and an orientation close to the C-orientation, respectively, with some local orientation scatter. At the grain boundary three bulges or new grains 1, 2 and 3 formed, which started to grow into the deformed grain $\mathbf{B}$ and whose orientations fall exactly in the range of those found in the deformed grain $\mathbf{A}$. Thus, it is concluded that the new recrystallized grains nucleated within the grain $\mathbf{A}$ and subsequently grew beyond the former grain boundary into grain $\mathbf{B}$ (Figure 9).

Figure 10a shows an ODF, which was computed from $~ 200$ examples of bulges or new SIBM-grains in the same sample. The texture strongly resembles the typical rolling texture of Al-alloys with fairly large orientation scatter, however. Hence, it is concluded that SIBM yields nucleus orientations close to the orientation spectrum of the rolling texture, but it reveals fairly large orientation scatter obviously reflecting the orientation gradients in the vicinity of the grain boundaries.

In the final recrystallization textures of Fe-containing Al-alloys, however, only the R-orientation (i.e. close to the S-orientation) occurs, but the other rolling texture orientations $\mathrm{B}$ and $\mathrm{C}$ of the $\beta$-fibre are usually missing. Thus, a further selection mechanism during the growth of the SIBM-grains is apparent. In order to elucidate this mechanism, the evolution of the local orientations of the SIBM-grains was followed with progressing recrystallization. In Figure 10b, the ODF of $\sim 250$ SIBM-grains at a later stage of recrystallization $(X(t) \approx 30 \%)$ is shown. As in Figure 10a, rolling texture orientations prevail, the orientations close to the B-orientation have almost vanished, however. Furthermore, the exact positions of the texture maxima in the various $\varphi_{2}-$ sections have remarkably shifted with respect to those in Figure 10a. Hence, it is concluded that during progressing recrystallization a growth selection of the R-oriented grains at the expense of the other rolling texture orientations $\mathrm{C}$ and $\mathrm{B}$ takes place. In comparison to the other rolling texture orientations, the R-orientation is distinguished by a preferred growth based on a $40^{\circ}<111>$-orientation relationship to the rolling texture 


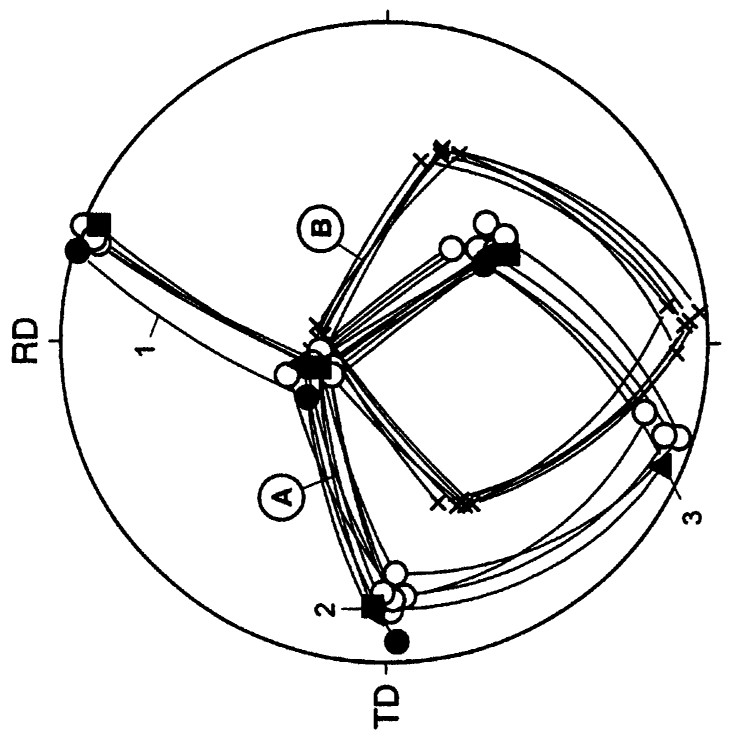

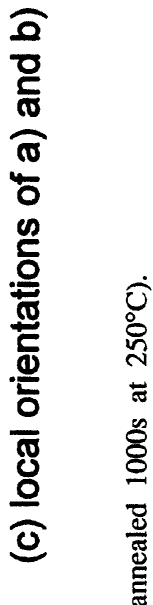

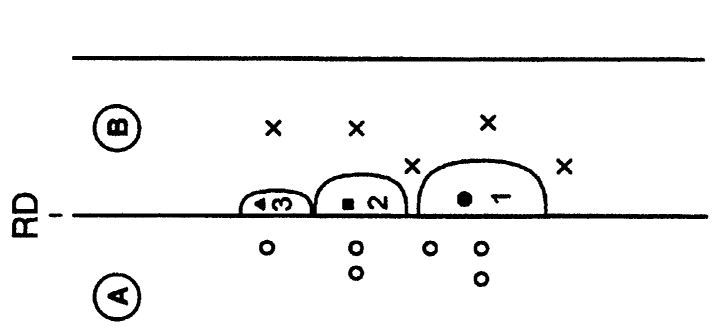

$\stackrel{0}{5}$

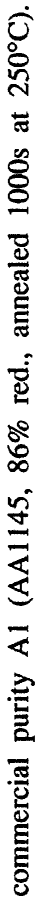

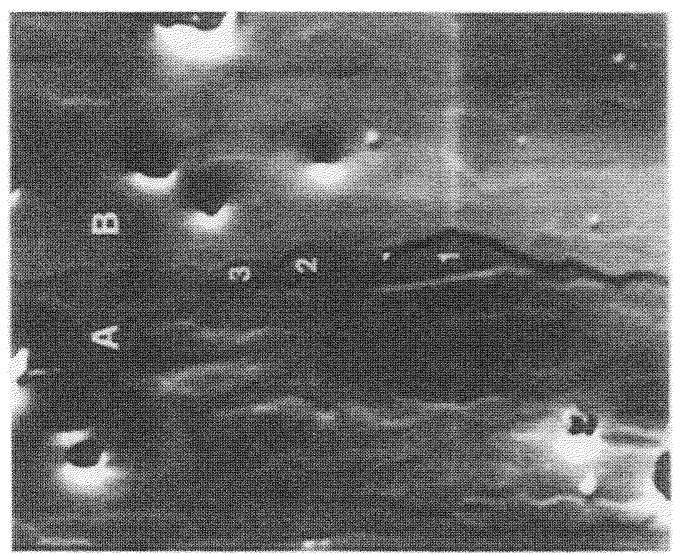

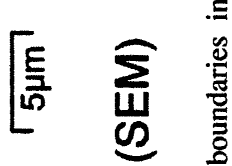

离造

인

过 $\frac{0}{\mathrm{E}}$

a

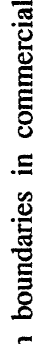

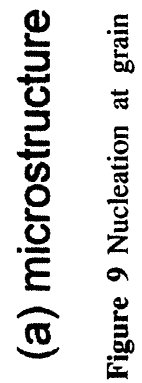



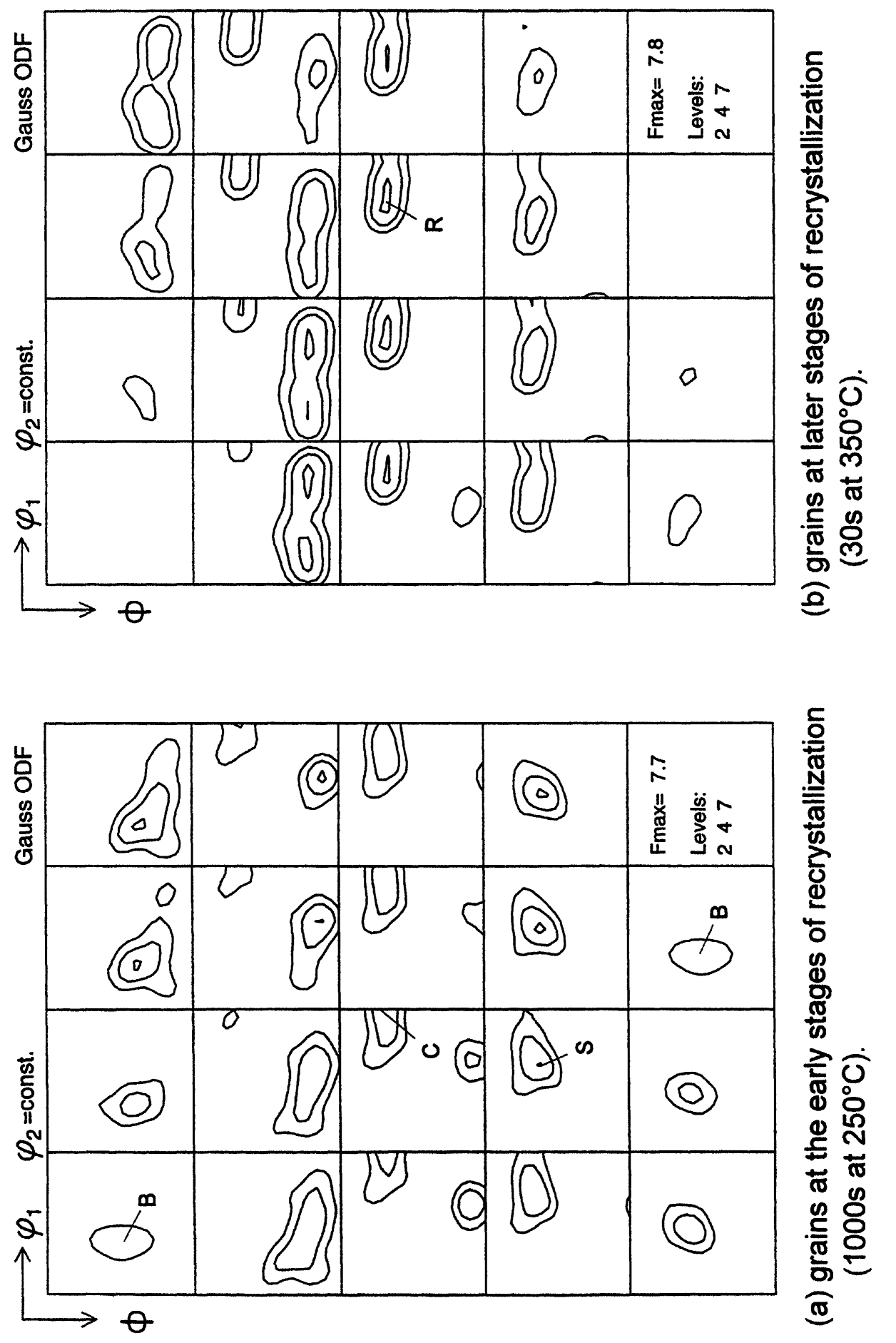

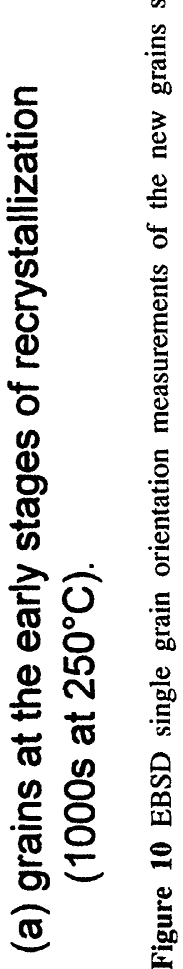


(Ito et al., 1983, Mao et al., 1988). As shown schematically in Figure 11, an approximate $40^{\circ}<111>$-orientation relationship is fulfilled between the R-orientation in the recrystallization texture (filled symbols) and each of the three remaining symmetrically equivalent components of the S-orientation in the rolling texture (open symbols). So it can be assumed that subgrains with a slightly scattered S-orientation close to the former grain boundaries are able to grow very easily into neighbouring grains, which exhibit one of the symmetrically equivalent S-components.

\section{CONCLUSIONS}

Single grain orientation measurements are conveniently performed with highest accuracy by evaluation of transmission Kikuchi diagrams in the TEM or backscattered Kikuchi diagrams in the SEM. For the investigation of bulk specimens, the EBSD technique in the SEM is best suited owing to its ease of use. If the spatial resolution accessible by $\operatorname{EBSD}(0.5 \mu \mathrm{m})$ is not sufficient, e.g. in case of deformed specimen, Kikuchi diagrams in the TEM can be obtained by MBED or, for highest spatial resolution, by CBED.

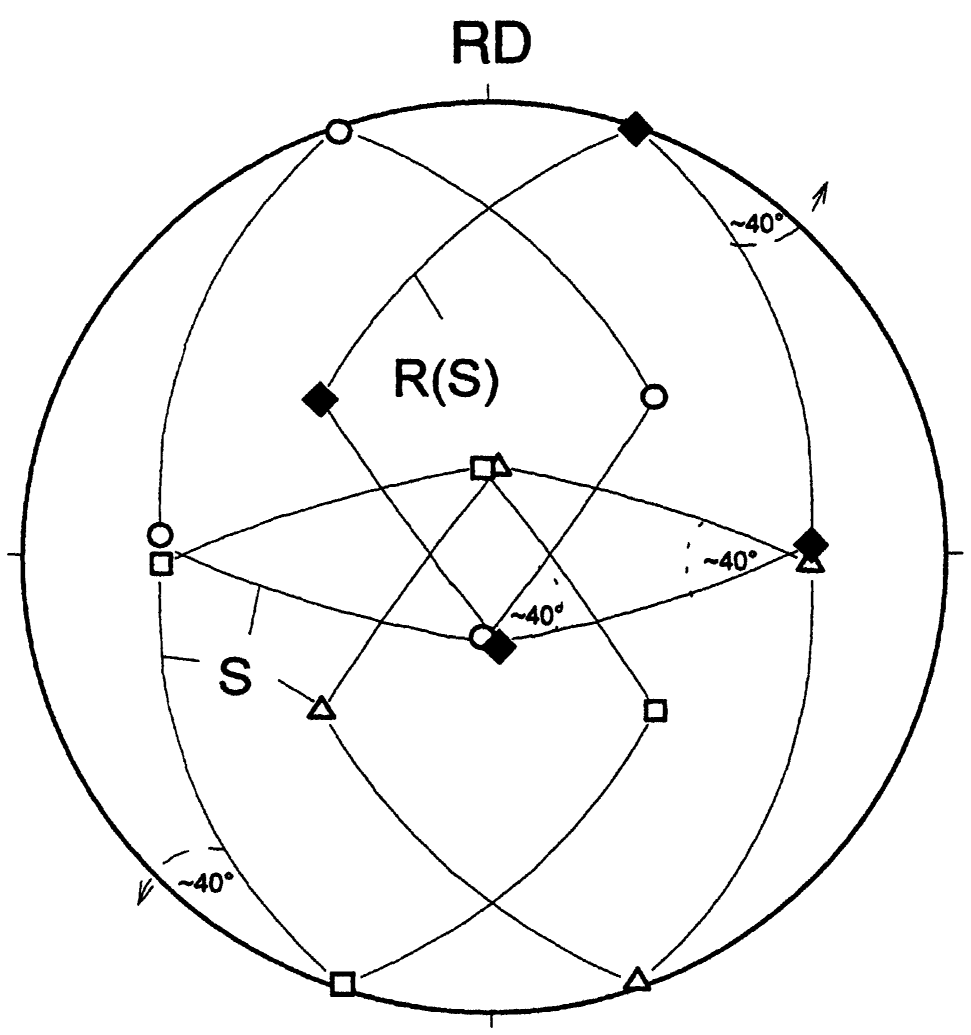

Figure $1140^{\circ}<111>$ orientation relationship between one component of the R-orientation (filled symbols) and the other three symmetrically equivalent components of the rolling texture S-orientation (open symbols). 
TEM Kikuchi diagrams and EBSD patterns follow the same crystallographic principles and, consequently both kinds of diagrams can be evaluated by similar procedures. The paper describes an algorithm for automated indexing and subsequent orientation determination, which is applied to both kinds of Kikuchi diagrams. For EBSD, the automated pattern recognition code yields very reliable results in virtually all cases and hence, in combination with an automated stage control, this enables one to carry out fully automated orientation microscopy. For automatic pattern recognition in TEM Kikuchi diagrams, more serious problems had to be overcome and in some cases interaction by the user is required.

The unique potential of these techniques for single grain orientation measurements is demonstrated by two examples pertaining to the microstructural evolution during the formation of recrystallization nuclei and their subsequent growth.

The incipient stages of recrystallization nucleation in cold rolled $\mathrm{Ni}_{3} \mathrm{Al}$ were analysed by means of in-situ annealing experiments in a TEM. In that instance, the fast online orientation determination helps to track orientation changes during the annealing process, as the development of new orientations can easily be recorded and the further evolution of the respective grains can be traced. Already at incipient nucleation twin relationships are observed, which leads to the conclusion that twinning seems to play a vital role in the formation of new grains in $\mathrm{Ni}_{3} \mathrm{Al}$.

The evolution of new grains which nucleate by SIBM at grain boundaries in cold rolled Al-alloys was tracked from the early stages of recrystallization through the later stages during growth of the nuclei. To yield statistically reliable data such experiments can only be performed by large scale EBSD measurements in a SEM. The results clearly evidence the occurrence of a selection mechanism of R-oriented grains to the disadvantage of other grains out of the orientation spectrum of the nuclei provided by the grain boundaries.

\section{Acknowledgements}

The authors like to thank R. Münch, A. Wiesweg, F. Lashgari and A. Lecki for their help in developing the computer codes used for the evaluation of the Kikuchi diagrams.

\section{References}

Adams, B. L., Wright, S. I. and Kunze, K. (1993). Metall. Trans., 24A, 819-831.

Bailey, J. E. (1960). Phil. Mag., 5, 833-842.

Ball, J. and Gottstein, G. (1993a). Intermetallics, 1, 191-208.

Ball, J. and Gottstein, G. (1993b). Intermetallics, 1, 171-185.

Beck, P. A. and Hu, H. (1952). Trans. AIME, 194, 83-90.

Beck, P. A. and Hu, H. (1966). "Recrystallization, Grain Growth and Textures", (ed. H. Margolin) A.S.M., Metals Park, Ohio, 393-433.

Bellier, S. P. and Doherty, R. D. (1977). Acta metall., 25, 521-538.

Dadson, A. B. C. and Doherty, R. D. (1992). Acta metall. mater., 40, 2053-2058.

Dingley, D. J. (1984). Scanning Electron Microscopy, 1984/II, 569-575.

Dingley, D. J. and Randle, V. (1992). J. Mater. Sci., 27, 4545-4566.

Dingley, D. J., Longden, M., Weinbren, J. and Alderman, J. (1987). Scanning Microscopy, 1, $451-456$.

Engler, O. and Gottstein, G. (1992). Steel research, 63, 413-418.

Engler, O., Gottstein, G., Pospiech, J. and Jura, J. (1994). Mat. Sci. Forum, 157-162, 259-274.

Engler, O., Vatne, H. E. and Nes, E. (1996). Mat. Sci. Eng., A205, 187-198.

Escher, C. and Gottstein, G. (1994). Mat. Sci. Forum., 157-162, 945-952.

Gerth, D. and Schwarzer, R. A. (1993). Textures and Microstructures, 21, 177-193.

Gottstein, G. (1988). Proc. ICOTOM 8 (eds. J. S. Kallend, G. Gottstein) TMS, Warrendale Pa, 195-202. 
Gottstein, G. (1992). Neue Hütte, 37, No. 4, 127-141.

Harland, C. J., Akhter, P. and Venables, J. A. (1981). J. Phys. E., 14, 175-182.

Hjelen, J. and Nes, E. (1990). Proc. XII Int. Congr. for Electron Microscopy, San Francisco Press, $404-405$.

Hjelen, J., Ørsund, R., Hoel, E., Runde P., Furu, T. and Nes, E. (1993). Textures and Microstructures, 20, $29-40$.

Høier, R., Bentdal, J., Daaland, O. and Nes, E. (1994). Mat. Sci. Forum, 157-162, 143-148.

Hough, P. V. C. (1962). US Patent 3069.654 Dec. 18.

Hu, H. (1963). "Recovery and Recrystallization of Metals", (ed. L. Himmel) Interscience Publ., New York, 311-378.

Hu, H. (1969). "Textures in Research and Practice", (eds. J. Grewen, G. Wassermann) Springer-Verlag, Berlin, 200-226.

Humphreys, F. J. (1984). Proc. 5th Risø Int. Symp. (eds. N. Hessel Andersen et al.), Risø Nat. Lab., Roskilde, 35-50.

Ito, K., Musick, R. and Lücke, K. (1983). Acta metall., 31, 2137-2149.

Juul Jensen, D. and Schmidt, N. H. (1990). Proc. Recrystallization '90 (ed. T. Chandra) TMS, Warrendale $\mathrm{Pa}, 219-224$.

Kikuchi, S. (1928). Jap. J. Phys., 5, 83-96.

Krieger Lassen, N. C. (1995). Proc. 16th Risø Int. Symp., (eds. N. Hansen et al.), Risø Nat. Lab., Roskilde, 405-411.

Krieger Lassen, N. C. and Bilde-Sørensen, J. B. (1993). J. of Microscopy, 170, 125-129.

Krieger Lassen, N. C., Juul Jensen, D. and Conradsen, K. (1992). Scanning Microscopy, 6, 115-121.

Krieger Lassen, N. C., Juul Jensen, D. and Conradsen, K. (1994). Mat. Sci. Forum, 157-162, 149-158.

Kunze, K., Wright, S. I., Adams, B. L. and Dingley, D. J. (1993). Textures and Microstructures, 20, 41-54.

Mao, W., Hirsch, J. and Lücke, K. (1988). Proc. ICOTOM 8, (eds. J.S. Kallend, G. Gottstein), TMS, Warrendale $\mathrm{Pa}, 613-618$.

Randle, V. (1992). Microtexture Determination and its Applications, The Inst. of Metals, London.

Schmidt, N. H., Bilde-Sørensen, J. B. and Juul Jensen, D. (1991). Scanning Microscopy, 5, 637-643.

Schwarzer, R. A. (1993). Textures and Microstructures, 20, 7-27.

Schwarzer, R. A., Zaefferer, S. and Kunze, K. (1995). Adv. in X-Ray Analysis, 38, 547-550.

v. Heimendahl, M., Bell, W. and Thomas, G. (1964). J. Appl. Phys., 35, 3614-3616.

Venables, J. A. and Harland, C. J. (1973). Phil. Mag., 27, 1193-1200.

Voss, K. and Süße, H. (1991). Praktische Bildverarbeitung, Carl Hanser Verlag, München, Wien.

Weiland, H. (1994). J. of Metals, 46, No. 9, 37-41.

Wright, S. I. and Adams, B. L. (1992). Metall. Trans., 23A, 759-767.

Wright, S. I., Zhao, J.-W. and Adams, B. L. (1991). Textures and Microstructures, 13, 123-131.

Young, C. T. and Lytton, J. L. (1972). J. Appl. Phys., 43, 1408-1417.

Zaefferer, S. and Schwarzer, R. A. (1994a). Z. Metallk., 85, 585-591.

Zaefferer, S. and Schwarzer, R. A. (1994b). Mat. Sci. Forum, 157-162, 247-250. 